\title{
CHARACTERIZATION AND ANTIMICROBIAL SUSCEPTIBILITY PROFILE OF NON LACTOSE FERMENTING GRAM-NEGATIVE BACTERIAL ISOLATES IN A TERTIARY CARE TEACHING HOSPITAL OF CENTRAL INDIA
}

\author{
ABHISHEK MEHTA ${ }^{1 *}$, MANISH KR DIWAKAR ${ }^{2}$ \\ ${ }^{1}$ Department of Microbiology, Government Medical College, Datia, Madhya Pradesh, India. ${ }^{2}$ Department of Microbiology, State Medical
} College, Shahjahanpur, Uttar Pradesh, India. Email: abhishekmehta623@gmail.com

Received: 30 June 2021, Revised and Accepted: 28 August 2021

\section{ABSTRACT}

Objectives: The non-lactose fermenting Gram-negative bacilli (NLF-GNB) are notorious pathogens reportedly acquiring multiple drug resistance alarmingly and emerging as a public health threat globally. This study was conducted to isolate and identify these pathogens from clinical samples received routinely in our Bacteriology Laboratory and to analyze their antibiotic susceptibility patterns.

Methods: In this cross-sectional study, the first 100 NLF-GNB strains isolated consecutively from 1218 clinical samples were included through convenience sampling. The samples were processed using standard microbiological techniques.

Results: The most common isolate was Pseudomonas aeruginosa followed by Acinetobacter spp, Proteus spp, Shigella spp, Salmonella typhi, Providencia spp., and Morganella spp. P. aeruginosa and Acinetobacter spp. isolates were found to exhibit high susceptibility toward Colistin and Imipenem. Proteus spp. exhibited high sensitivity toward Imipenem, Aminoglycosides, Ceftazidime, and Cefepime. All Providencia isolates were susceptible to Amikacin, Cefepime, and Ceftriaxone. The only isolate of Morganella spp. was found to be susceptible to Amikacin, Cefepime, Ceftazidime, Piperacillin tazobactam, Ciprofloxacin, Imipenem, and Aztreonam. Shigella isolates exhibited very high susceptibility toward Imipenem followed by Gentamicin and Ceftazidime. All the isolates of $S$. typhi exhibited susceptibility toward Imipenem, Piperacillin tazobactam, Ceftazidime, Ceftriaxone, Cefoperazone sulbactam, and Chloramphenicol. 24\% of test isolates were found to be Multidrug resistant.

Conclusion: Antimicrobial surveillance is needed to implement appropriate timely interventions to restrict the spread of multidrug-resistant clones. Strict infection prevention and control practices, with judicious antibiotic prescription policy, may help in tackling this problem by obviating the selection pressure.

Keywords: Non-lactose fermenting Gram-negative bacilli, Antimicrobial susceptibility, Antibiotic resistance, Samples, Isolates, Multidrug resistance. (c) 2021 The Authors. Published by Innovare Academic Sciences Pvt Ltd. This is an open access article under the CC BY license (http://creativecommons.org/ licenses/by/4.0/) DOI: http://dx.doi.org/10.22159/ajpcr.2021v14i10.42822. Journal homepage: https://innovareacademics.in/journals/index.php/ajpcr

\section{INTRODUCTION}

Aerobic non-fermenting Gram-negative bacilli (NFGNB) are a taxonomically diverse group of bacteria that are either not capable of utilizing carbohydrates as an energy source or degrading them via oxidative pathway [1-3].

NFGNB (Pseudomonas, Acinetobacter, Burkholderia, Stenotrophomonas, etc.) constitute about $15 \%$ of all clinical bacterial isolates. NFGNBs are emerging as important health-care-associated pathogens in the current scenario. Hospital strains are found to exhibit multidrug resistance (MDR). They have been incriminated in infections, such as septicemia, meningitis, pneumonia, urinary tract infections, and surgical site infections. NFGNBs are innately resistant to many antibiotics and are known to produce extended-spectrum ß-lactamases (ESBL) and Metallo ß-lactamases [4-6].

Apart from NFGNB, there is another group of non-lactose fermenters capable of utilizing glucose (Proteus, Salmonella, Shigella, Morganella, Providencia, etc.). These NLF-GNB are Notorious pathogens reportedly acquiring multiple drug resistance alarmingly and emerging as a public health threat.

MDR has been emerging rapidly and consistently in these groups of pathogens driven by selection pressure due to inappropriate irrational drug therapy. This has resulted in treatment failures leading to an extended hospital stay, health complications, and a significant rise in morbidity and mortality. The study of antibiotic susceptibility patterns of isolates is imperative in formulating a strategy for prompt and appropriate therapy and also plays a crucial role in the prevention and control of the disease. Continual consistent surveillance and monitoring of local antimicrobial resistance trends is a prerequisite for implementing rational measures and updating the therapeutic guidelines [4,6-8].

There are very few studies from India wherein the various NLF-GNBs, isolated from patients' samples, have been identified and their clinical significance assessed. Hence, this cross-sectional study was undertaken to identify the various non-lactose fermenters isolated from the patients attending our hospital to assess their clinical significance and antimicrobial susceptibility pattern.

\section{METHODS}

This cross-sectional study was conducted in the Department of Microbiology, Government Medical College, Datia (MP), India, after obtaining clearance from Institutional Ethics Committee.

\section{Study period and clinical samples}

Samples such as blood, urine, sputum, wound swabs, pus, stool, other body fluids, ear swabs, throat swabs, and nasal swabs obtained from patients admitted or attending OPDs of various clinical departments in GMC, Datia, submitted to the Department of Microbiology for routine diagnostic workup.

\section{Sample size}

Through convenience sampling, first 100 non-lactose fermenting Gram-negative bacterial strains isolated consecutively from the clinical 
samples processed as per the standard protocol in the duration between October 1, 2018, to April 30, 2020, whichever is earlier were included in the study.

The NLF-GNB isolates found to be contaminants or commensals concerning for the respective samples were excluded from the study. For e.g., Pseudomonas aeruginosa or Proteus spp. if isolated from stool culture were not included in the study, being a part of commensal flora of gastrointestinal tract.

Isolation and identification of bacterial isolates were done using standard microbiological techniques. The antimicrobial susceptibility test of all the test isolates was performed by the Kirby-Bauer Standard disc diffusion method and results were interpreted according to the CLSI 2018 guidelines [9-11].

\section{RESULTS AND DISCUSSION}

Through convenience sampling, first 100 non-lactose fermenting Gram-negative bacterial strains were isolated consecutively from 1218 clinical samples, starting from October 1, 2018. The study target was achieved till January 31, 2020 (16 months).

The majority of samples yielding NLF-GNB test isolates belonged to the patients in the age group of $40-60$ years (42\%) followed by the patients in the age group of $20-39$ years $(34 \%)$.

Similar findings were observed in the study conducted by Grewal et al. 2020, Benachinmardi et al. 2020, Reddy et al. 2019, Sajjad et al. 2017, and Bohra et al. 2017. However, Akbar et al. 2014 in a similar study conducted in Peshawar, Pakistan, found that most of the test isolates were contributed by the females and patients in the 11-30 years age group [1,8,12-14].

As evident in Fig. 1, more than half of the NLF test isolates were obtained from pus and urine. This finding is in concordance with other similar studies conducted by Reddy et al. 2019, Bhargava et al. 2015, Akbar et al. 2014, Malini et al. 2009, and Grewal et al. 2020. But in the majority of studies conducted in the recent past, the test isolates were derived predominantly from pus and respiratory samples $[1,7,12,15,16]$.

As evident in Fig. 2, the most common test organism isolated was P. aeruginosa followed by Acinetobacter spp. This finding is as per the similar studies by Upgade et al. 2012, Nautiyal et al. 2014, Gore and Pai 2015, Bhargava et al. 2015, Kamalraj et al. 2015, Mahajan et al. 2016, Sajjad et al. 2017, Gunasekar et al. 2018, Nazir et al. 2019, Reddy et al. 2019, Benachinmardi et al. 2020, Grewal et al. 2020, and Juyal et al. 2020 [1,2,5,7,8,12,13,17-21].

But unlike our study, in few similar studies conducted by Sharma and Pant, 2017, Shah and Vaghela, 2018, and Paul and Borah, 2020, Acinetobacter spp. was the most common isolate followed by $P$. aeruginosa $[4,22,23]$.

Out of the 100 samples yielding the test isolates, polymicrobial growth was seen in 22 samples where the NLF-GNB were isolated along with other organisms. None of the samples yielded more than one NLF isolate.

The overall isolation rate of NLF isolates from the total 1218 clinical samples processed came out to be $8.2 \%$. P. aeruginosa (4\%) exhibited the highest isolation rate followed by Acinetobacter (2\%). The samplewise and organism-wise isolation rates are depicted in Tables 1 and 2.

As evident in Table 3, $P$ aeruginosa was more common in pus, ear swabs, and urine contributing to $80 \%$ of its isolates, while less common in blood, body fluids, sputum, and throat swabs. This finding is in concordance with other similar studies conducted by Reddy et al. 2019, Bhargava et al. 2015, Akbar et al. 2014, Malini et al. 2009, and Grewal et al. $2020[1,7,12,15,16]$.

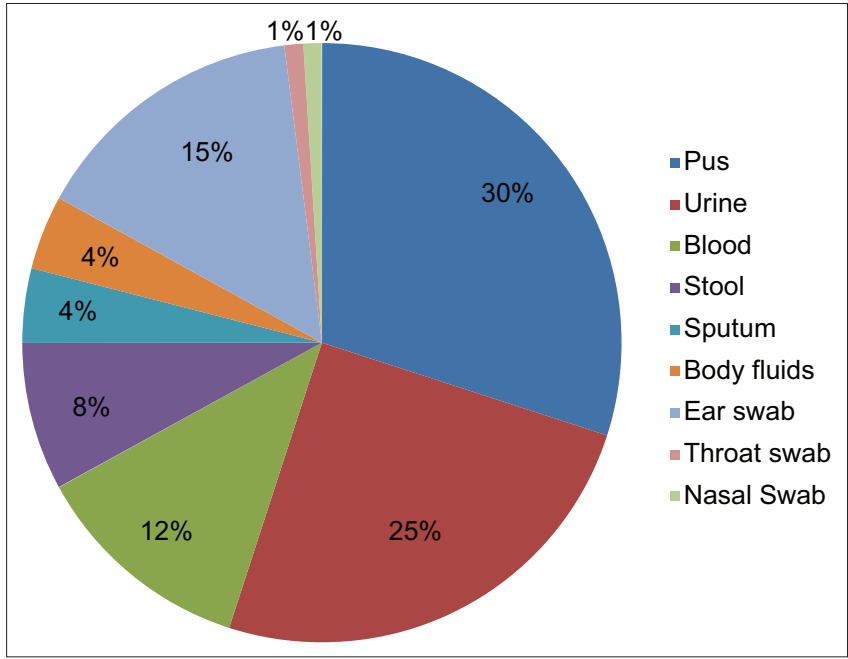

Fig. 1: Sample wise distribution of NLF isolates

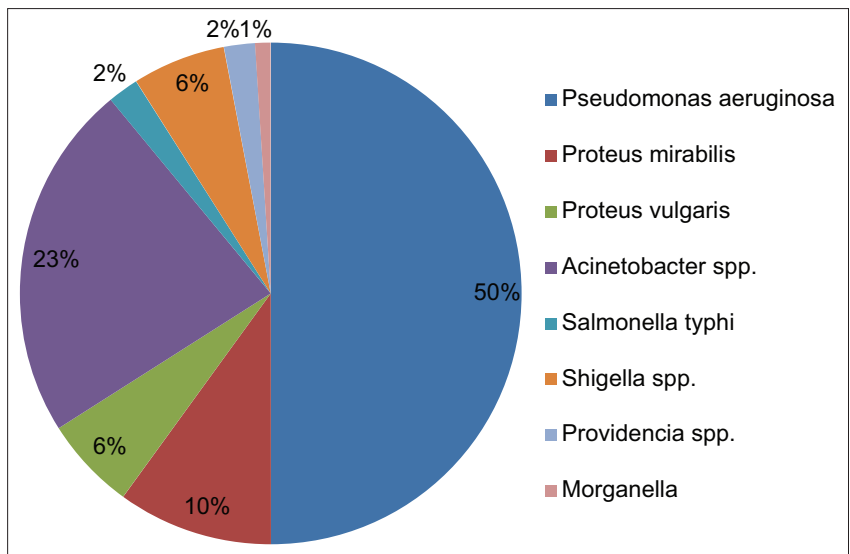

Fig. 2: Organism-wise distribution of non-lactose fermenting isolates

Table 1: Sample-wise isolation rate of test isolates

\begin{tabular}{lllll}
\hline S. No. & Specimens & $\begin{array}{l}\text { Number of } \\
\text { samples }\end{array}$ & No. of NLF isolates & $\begin{array}{l}\text { \% } \\
\text { isolation }\end{array}$ \\
\hline 1. & Pus & 450 & 30 & 6.67 \\
2. & Urine & 438 & 25 & 6 \\
3. & Blood & 114 & 13 & 11.4 \\
4. & Stool & 66 & 08 & 12 \\
5. & Sputum & 56 & 04 & 7 \\
6. & Body fluids & 46 & 04 & 9 \\
7. & Throat swab & 28 & 01 & 3.6 \\
8. & Nasal Swab & 20 & 01 & 5 \\
& Total & 1218 & 100 & 8.2 \\
\hline
\end{tabular}

Table 2: Organism-wise isolation rate of non lactose fermenting isolates

\begin{tabular}{llll}
\hline S. No. & Organism & No. of isolates & \% isolation \\
\hline 1. & Pseudomonas aeruginosa & 50 & 4 \\
2. & Proteus mirabilis & 10 & 0.8 \\
3. & Proteus vulgaris & 05 & 0.4 \\
4. & Acinetobacter spp. & 23 & 2 \\
5. & Salmonella typhi & 03 & 0.25 \\
6. & Shigella sp & 06 & 0.5 \\
7. & Providencia spp. & 02 & 0.16 \\
8. & Morganella spp. & 01 & 0.08 \\
\hline
\end{tabular}


Table 3: Sample-wise distribution of non-lactose fermenting isolates

\begin{tabular}{|c|c|c|c|c|c|c|c|c|c|c|c|}
\hline \multirow[t]{2}{*}{ S. No. } & \multirow[t]{2}{*}{ Non-lactose fermenting Isolates } & \multicolumn{10}{|c|}{ Samples } \\
\hline & & Pus & Urine & Blood & Stool & Sputum & Ear swab & Body fluids & Throat swab & Nasal Swab & Total \\
\hline 1. & Pseudomonas aeruginosa & 18 & 9 & 4 & - & 2 & 13 & 3 & 1 & - & 50 \\
\hline 2. & Proteus mirabilis & 4 & 5 & - & - & - & 1 & - & - & - & 10 \\
\hline 3. & Proteus vulgaris & 1 & 3 & 1 & - & - & - & - & - & - & 05 \\
\hline 4. & Acinetobacter spp. & 7 & 6 & 6 & - & 1 & 1 & 1 & - & 1 & 23 \\
\hline 5. & Salmonella typhi & - & - & 1 & 2 & - & - & - & - & - & 03 \\
\hline 6. & Shigella spp. & - & - & - & 6 & - & - & - & - & - & 06 \\
\hline 7. & Providencia spp. & - & 1 & 1 & - & - & - & - & - & - & 02 \\
\hline \multirow[t]{2}{*}{8.} & Morganella spp. & - & 1 & - & - & - & - & - & - & - & 01 \\
\hline & Total & 30 & 25 & 13 & 08 & 03 & 15 & 04 & 01 & 01 & 100 \\
\hline
\end{tabular}

In the present study, Acinetobacter spp. was found to be more common in pus, blood, and urine contributing to $83 \%$ of its isolates while less common in body fluids, sputum, ear swabs, and throat swabs. Similar findings were reported by Grewal et al. 2020, Reddy et al. 2019, and Kamalraj et al. 2015 [1,5,12] But in some studies by Malini et al. 2009, De Francesco et al. 2013, Benachinmardi et al. 2020, Sharma and Pant 2017, the organism was predominantly isolated from respiratory samples $[4,8,16,24]$.

In the present study, most of the isolates of Proteus spp. (87\%) were obtained from pus and urine like the other similar studies by Sharma and Pant, 2017, Wang et al. 2014, and Leulmi et al. 2014 [4,25,26] However, Bahashwan 2013 found that majority of Proteus spp. isolates were derived from sputum and wound swabs [27].

Out of three isolates of Salmonella typhi, two were obtained from stool culture and one from blood culture.

Shigella spp. being an enteropathogen, fecal specimens are the most preferred samples for culture. In our study too, all the six Shigella spp. isolates were obtained from stool culture as in the majority of similar studies [28-33].

Out of two isolates of Providencia spp., one each was isolated from urine and blood cultures. In the study period, we reported only one isolate of Morganella spp. from a urine sample.

In a study by Leulmi et al. 2014, Providencia spp. was mainly isolated from pus and urine cultures [26]. Liu et al. 2020, isolated Providencia spp. mainly from sputum and wound swabs [34].

In similar studies, Morganella spp. was predominantly isolated from pus, urine, and blood by Akbar et al. 2014, from pus and urine by Leulmi et al. 2014, and from urine by Sharma and Pant $2017[4,15,26]$.

As evident in Fig. 3, the highest number of test isolates were obtained from clinical specimens from the ENT department followed by the Surgery, Pediatric, and Medicine departments. $60 \%$ of the NLF test isolates were obtained from IPD patients and $40 \%$ from OPD patients, while an opposite trend was seen in the samples from ENT department, wherein contribution from OPD patients $(83 \%)$ far exceeded the IPD patients. On the other hand in the case of the Pediatric department, only 2 out of 18 isolates were obtained from OPD samples, with Pediatric ICUs (PICU and SNCU) contributing more than $60 \%$ of the test isolates.

The antibiotic susceptibility pattern of NLF test isolates is depicted in Table 4. In our study, P. aeruginosa isolates were found to exhibit high susceptibility toward Colistin and Imipenem and very less susceptibility toward Ciprofloxacin, Aztreonam, and Co-trimoxazole. Similar findings were reported by Shah and Vaghela, Sharma and Pant, and Bhargava et al. $[4,7,22]$.

Grewal et al. reported high resistance toward Aminoglycosides, Amoxycillin clavulanate, Fluoroquinolones, Aztreonam, and third and fourth generation Cephalosporins [1].

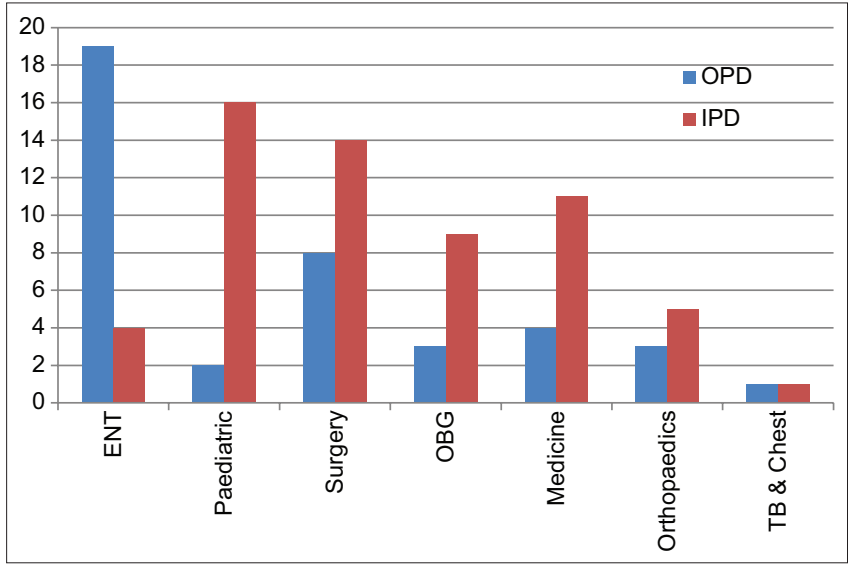

Fig. 3: Department-wise distribution of test isolates

Kamalraj et al. reported high susceptibility toward Imipenem and high resistance toward third-generation Cephalosporins (3GC) and Ciprofloxacin. They reported $38.3 \%$ and $42.3 \%$ ESBL strains, respectively [5].

Kamalraj et al., Nagaveni et al., and Anuradha et al. reported $11.6 \%, 24 \%$, and $28 \%$ of MBL producing strains of P. aeruginosa, respectively $[5,35,36]$.

Malini et al. and Gore and Pai reported high susceptibility toward Imipenem, Ticarcillin, Amikacin, and Cefoperazone and least toward Co-trimoxazole $[2,16]$.

Juyal et al. and Benachinmardi et al. reported high sensitivity toward Amikacin, Imipenem, Piperacillin tazobactam, and Ticarcillin clavulanate and least sensitivity toward Co-trimoxazole $[6,8]$.

In our study, Acinetobacter spp. isolates were found to exhibit high susceptibility toward Colistin and Imipenem and very less susceptibility toward Ciprofloxacin, Cefepime, Ceftriaxone, Aztreonam, Amoxycillin clavulanate, Gentamycin, Tobramycin, and Co-trimoxazole.

Malini et al. reported high sensitivity toward Imipenem and Piperacillin and low susceptibility toward Ciprofloxacin, Co-trimoxazole, and third and fourth generation Cephalosporins [16]. Nautiyal et al. reported high sensitivity toward Imipenem and Piperacillin tazobactam and low sensitivity toward Aminoglycosides, Fluoroquinolones, 3GC, and Cotrimoxazole [18].

Grewal et al. reported high sensitivity toward Imipenem and Cefoperazone sulbactam and low sensitivity toward Polymixin group, Ureidopenicillin group, Amoxycillin clavulanate, Aminoglycosides, Fluoroquinolones, Aztreonam, and third and fourth generation Cephalosporins [1]. 
Table 4: Antibiotic susceptibility pattern of test isolates

\begin{tabular}{|c|c|c|c|c|c|c|c|c|}
\hline \multirow[t]{2}{*}{ S. No } & \multirow[t]{2}{*}{ Antibiotics } & \multicolumn{7}{|c|}{ No. of susceptible isolates n (\%) } \\
\hline & & $\begin{array}{l}\text { Pseudomonas } \\
\text { aeruginosa }\end{array}$ & $\begin{array}{l}\text { Proteus mirabilis } \\
\text { Proteus vulgaris }\end{array}$ & $\begin{array}{l}\text { Acinetobacter } \\
\text { spp. }\end{array}$ & $\begin{array}{l}\text { Salmonella } \\
\text { typhi }\end{array}$ & $\begin{array}{l}\text { Shigella } \\
\text { sp. }\end{array}$ & $\begin{array}{l}\text { Providencia } \\
\text { sp. }\end{array}$ & $\begin{array}{l}\text { Morganella } \\
\text { sp. }\end{array}$ \\
\hline 1. & Amikacin (30) & $30(60)$ & $13(86.67)$ & $13(56.5)$ & $2(66.7)$ & $4(66.7)$ & $2(100)$ & 1 \\
\hline 2. & Gentamicin (10) & $25(50)$ & $13(86.67)$ & $10(43.5)$ & $1(33)$ & $5(83)$ & 0 & 0 \\
\hline 3. & Cefepime $(30)$ & $30(60)$ & $12(80)$ & $9(39)$ & $2(66.7)$ & $2(33)$ & $2(100)$ & 1 \\
\hline 4. & Ceftazidime (30) & $32(64)$ & $11(73.3)$ & $11(48)$ & $3(100)$ & $5(83)$ & $1(50)$ & 1 \\
\hline 5. & Ceftriaxone (30) & $24(48)$ & $10(66.67)$ & $10(43.5)$ & $3(100)$ & $4(66.7)$ & $2(100)$ & 0 \\
\hline 6. & $\begin{array}{l}\text { Cefoperazone } \\
\text { sulbactum }\end{array}$ & $23(46)$ & $6(40)$ & $11(48)$ & $3(100)$ & $5(83)$ & $1(50)$ & 0 \\
\hline 7. & $\begin{array}{l}\text { Amoxycillin } \\
\text { clavulanate }\end{array}$ & $27(54)$ & $7(46.67)$ & $10(43.5)$ & $2(66.7)$ & $4(66.7)$ & 0 & 0 \\
\hline 8. & $\begin{array}{l}\text { Piperacillin } \\
\text { tazobactam }\end{array}$ & $34(68)$ & 7 (46.67) & $12(52)$ & $3(100)$ & $5(83)$ & 0 & 1 \\
\hline 9. & Ciprofloxacin (5) & $23(46)$ & $7(46.67)$ & $7(30)$ & $2(66.7)$ & $5(83)$ & $1(50)$ & 1 \\
\hline 10. & $\begin{array}{l}\text { Trimethoprim } \\
\text { sulfamethoxazole }\end{array}$ & $19(38)$ & $5(33.3)$ & $6(26)$ & $2(66.7)$ & $2(33)$ & $1(50)$ & 0 \\
\hline 11. & Imipenem (10) & $42(84)$ & $15(100)$ & $19(82.6)$ & $3(100)$ & $6(100)$ & $1(50)$ & 1 \\
\hline 12. & Aztreonam & $13(26)$ & - & $3(13)$ & - & - & 0 & 1 \\
\hline 13. & Colistin & $50(100)$ & - & $22(95.6)$ & - & - & - & 0 \\
\hline 14. & Chloramphenicol & - & $8(53.3)$ & - & $3(100)$ & $3(50)$ & 0 & 0 \\
\hline 15. & Tetracyclin & - & - & - & - & $2(33)$ & 0 & - \\
\hline 16. & Tobramycin & $28(56)$ & $12(80)$ & $10(43.5)$ & - & - & $1(50)$ & - \\
\hline
\end{tabular}

A very high level of resistance to different groups of antibiotics was reported by Benachinmardi et al., Juyal et al., Gupta et al., and Hodiwala et al., but the strains were comparatively susceptible to Imipenem $[6,18,37,38]$.

Uma et al. 2009 and Anil et al. 2011 reported 70.9\% and 21\% of MBL producing strains of Acinetobacter baumannii, respectively [39,40]. Kamalraj et al. and Sinha et al. reported $25 \%$ and $28 \%$ of ESBL strains, respectively $[5,41]$.

A. baumannii is gaining more importance as a nosocomial notorious pathogen due to its potential to form a biofilm, which accounts for its outstanding antibiotic resistance and high virulence.

Proteus spp. in the present study exhibited high sensitivity towards Imipenem, Tobramycin, Amikacin, Gentamicin, Ceftazidime, and Cefepime and less sensitivity toward Cefoperazone sulbactam, Amoxycillin clavulanate, Piperacillin tazobactam, Ciprofloxacin, and Cotrimoxazole.

Proteus mirabilis strains are generally found to be more susceptible to antimicrobials than Proteus vulgaris and other Proteus species. P. mirabilis has got intrinsic resistance to nitrofurantoin and tetracycline which could be used as an identification marker. However, it is generally susceptible to the Carbapenems, Penicillins, Aminoglycosides, and Cotrimoxazole [42].

Bahashwan 2013 reported high sensitivity of Proteus spp. isolates toward Imipenem followed by Amikacin, Cefoxitin, Aztreonam, and Piperacillin. The other antibiotics (Amoxycillin clavulanate, Gentamicin, Ceftazidime, Ciprofloxacin, Cephalothin, Cefpiramide, and Co-trimoxazole) exhibited $<40 \%$ sensitivity [27].

Feglo et al. 2010 reported very high levels of resistance in Proteus spp. isolates against Ampicillin, Co-trimoxazole, Tetracycline, and Chloramphenicol [43].

Mirzaei et al. reported high sensitivity in urinary isolates of $P$. mirabilis toward Amoxicillin-clavulanate, Carbapenems, 3GC, Aztreonam, Tobramycin, and Fluoroquinolones and low sensitivity toward Cotrimoxazole and Amoxicillin. They found all isolates exhibiting the capability of biofilm formation with $72 \%$ of strains being strong biofilm producers. $24 \%$ of isolates were sensitive to all antibiotics tested, while one isolate was pan resistant. $14.5 \%$ of the isolates were MDR strains and $10 \%$ were ESBL producers. Most of the ESBL producers were MDR strains [44].

Swenson et al. 1999 have shown that all P. vulgaris and Proteus penneri strains have got the capability of producing inducible $\beta$-lactamases which could hydrolyze primary and extended-spectrum penicillins and cephalosporins. This calls for the need for monitoring the drug susceptibility of Proteus isolates [45].

Since we have only one Morganella spp. and two Providencia spp. isolated in the present study, any statistically significant inference could not be drawn from the existing study data regarding antimicrobial resistance.

The only isolate of Morganella spp. was found to be susceptible to Amikacin, Cefepime, Ceftazidime, Piperacillin tazobactam, Ciprofloxacin, Imipenem, and Aztreonam and resistant to Gentamicin, Ceftriaxone, Cefoperazone sulbactam, Amoxycillin clavulanate, Trimethoprimsulfamethoxazole, Colisin, and Chloramphenicol.

All Providencia isolates were found to be susceptible toward Imipenem, Amikacin, Cefepime, and Ceftriaxone, while none toward Gentamycin, Amoxycillin clavulanate, Piperacillin tazobactam, Aztreonam, Chloramphenicol, and Tetracyclin.

Lieu et al. reported high susceptibility of Providencia stuarti isolates toward Imipenem, Fluoroquinolones, Amikacin, and Cefepime. All test isolates were resistant to Ampicillin sulbactam, Amoxicillin clavulanate, Ticarcillin clavulanate, Piperacillin tazobactam, Aztreonam, and 3GC. All 76 isolates were found to be ESBL producers out of which $92 \%$ exhibited multiple drug resistance [34].

In hospital settings, high levels of Ciprofloxacin resistance have been reported frequently for the Proteus and Providencia spp. isolates due to rampant usage of the same. Fass et al. 1995 reported decreasing susceptibility of $P$. stuartii to ciprofloxacin from $100 \%$ to $46 \%$ over a 6 year period [46].

P. penneri is generally found to be more resistant to penicillins than $P$. vulgaris, and its susceptibility pattern resembles more with Morganella morganii than with other Proteus spp. 
M. morganii and P. penneri are generally susceptible to cefoxitin, 3GC,4GC, aztreonam, aminoglycosides, ciprofloxacin, and imipenem and resistant to cefazolin, 1GC, 2GC, Cefoperazone, amino, and ureidopenicillins.

Leulmi et al. reported high sensitivity of Proteus, Providencia, and Morganella spp. isolates toward cefoxitin, cefotaxime, imipenem, amikacin, and ciprofloxacin, and very little sensitivity toward amoxicillin, nalidixic acid, and Co-trimoxazole [26].

M. morganii and P. stuartii isolates also exhibited high resistance to ticarcillin, gentamicin, and chloramphenicol. More than $61 \%$ of isolates were Multidrug resistant strains (resistant to at least 3 groups of antibiotics). $15 \%$ of the isolates were ESBL producers with no significant difference among various species.

In the present study, Shigella spp. isolates exhibited very high susceptibility toward Imipenem followed by Gentamicin, Ceftazidime, Cefoperazone sulbactam, Piperacillin tazobactam, and Ciprofloxacin and very less susceptibility toward Cefepime and Co-trimoxazole.

While comparing the resistance patterns between two studies by Mamtha et al. in 2007 and 2012 in the same region, all Shigella isolates were found to be resistant to nalidixic acid and a marked increase in resistance was observed. Resistance to ciprofloxacin increased from $30 \%$ to $87 \%$, norfloxacin from $20 \%$ to $83 \%$, ampicillin from $63 \%$ to $100 \%$, tetracycline from $74 \%$ to $84 \%$, and Co-trimoxazole from $79 \%$ to $90 \%$. However, there was a significant decrease in resistance against gentamicin and amikacin from $71 \%$ to $40 \%$ and $45 \%$ to $5 \%$, respectively [32,47].

In both the studies, Shigella isolates exhibited high sensitivity toward 3GC which is in accordance with our study. Similar findings were reported by Srinivasa et al. 2009 [31].

Shigellae may be susceptible to the aminoglycosides in vitro, but not in vivo due to poor penetration of the intestinal mucosa when given orally [48].

In addition to some fluoroquinolones, pivmecillinam (amdinocillin pivoxil) and ceftriaxone are the only antimicrobials found to be effective in the treatment of MDR strains of Shigella in all age groups. However, Azithromycin can be considered as an alternative drug among adults. However, these antibiotics should be used only when local strains are resistant to Ciprofloxacin [48].

Pazhani et al., in a study on childhood diarrhea (2001-2004), found that $50 \%$ of Shigella isolates were resistant to ampicillin and $96 \%$ to co-trimoxazole in 2001 , which was reduced to $32 \%$ and $83 \%$, in 2002. While $83 \%$ of isolates were resistant to tetracycline and $56 \%$ to nalidixic acid in 2001 which increased to $89 \%$ and $62 \%$, respectively, in 2002. In addition, fluoroquinolone resistance emerged among Shigella dysenteriae and Shigella flexneri isolates in 2002 increased gradually during the study period from $11 \%$ to $25 \%$ [29].

We have isolated only three $S$. typhi isolates in our study which is not sufficient to give a clear generalizable picture of the local antimicrobial resistance pattern in the organism. However, all the isolates of S. typhi exhibited susceptibility toward Imipenem, Piperacillin tazobactam, Ceftazidime, Ceftriaxone, Cefoperazone sulbactam, and Chloramphenicol. High levels of resistance were observed toward Gentamicin.

Similar findings were reported by Mehta et al. 2018 Choudhary et al. 2013, Kumar et al. 2013, Singhal et al. 2014, and Gurung et al. 2017 [49-53].

Mehta et al. 2018 and Gurung et al. 2017 reported MDR toward first-line drugs in more than $15 \%$ of $S$. typhi strains which were also resistant to Nalidixic acid $[49,53]$.
It is observed that many of the isolates resistant to Nalidixic acid (NARST) were found to exhibit in vitro susceptibility to fluoroquinolones. But as per CLSI guidelines, such strains (NARST) should be considered Fluoroquinolone resistant as Nalidixic acid is a surrogate marker to predict fluoroquinolone treatment failure.

The resistance against fluoroquinolones like Ciprofloxacin is following an increasing trend due to the selective pressure by its unrestricted injudicious usage in typhoid therapy. Nalidixic acid resistance among Salmonella spp. is rapidly increasing in India. However, the consistent use of Ciprofloxacin as the mainstay treatment of typhoid in NA resistant cases has led to a steady rise in MIC along with further mutations at the same locus which has resulted in the emergence of completely resistant strains.

Several studies in the recent past have shown a re-emergence of susceptibility of $S$. typhi toward the first-line antibiotics. This could be due to their inconsistent usage by clinicians over the last decade who are preferring newer antimicrobials over them resulting in the withdrawal of selection pressure.

In the present study, $24 \%$ of NLF-GNB test isolates were found to be Multidrug-resistant. In a hospital setting, Multidrug-resistant or panresistant strains may transmit from one patient to another through the hands of health-care workers or via environmental contamination. These notorious pathogens are the potential reservoirs of resistance genes that could be transferred to other bacterial strains. The high levels of $\beta$-lactamase production and multidrug resistance of the isolates is an emerging public health threat globally. Environmental surveillance, searching for asymptomatically colonized persons through screening of patients as well as health-care workers, and using molecular epidemiology should be the requisite strategy for investigating the clusters of infection with pan-resistant organisms. These wild strains have great potential to survive in the hospital environment so improved antibiotic stewardship and infection control measures will be needed to inhibit the emergence and spread of multidrug-resistant NFGNB in the health-care setting $[27,43,54]$.

In the current scenario due to rapidly emerging multiple drug resistance, the polymixins (polymyxin B and colistin) are being used frequently as the last line therapeutic option. However, the clinicians should restrict the use of the reserve drugs in exceptional conditions only [19].

\section{CONCLUSION}

The present study highlighted the fact that non-fermenter Gramnegative bacilli like P. aeruginosa and Acinetobacter spp. along with the other non-lactose fermenter, GNBs like the Proteae family, S. typhi, and Shigella spp. had emerged as important pathogens causing serious infections in the community as well as hospital settings. Infections caused by these notorious pathogens are difficult to treat as they are rapidly acquiring resistance to the commonly used antibiotics. Ever-increasing problem of antibiotic resistance has been worsened by the slow pace of research regarding the development of newer antimicrobial molecules.

Antimicrobial surveillance is needed to implement appropriate timely interventions to restrict the spread of multidrug-resistant clones. Strict infection prevention and control practices, with judicious antibiotics prescription policy, may help in tackling the emerging threat of multiple drug-resistant bugs by obviating the selection pressure. Appropriate judicious selection and rotation/cycling of antibiotics guided by the knowledge of their susceptibility profiles is of utmost importance.

The implementation of an antibiotic policy at the hospital level for the control and restriction of injudicious antimicrobial use is imperative in managing nosocomial infections.

This is the need of the hour to develop therapeutic protocols guided by susceptibility profiles for tuning antibiotic therapy regimens 
to minimize the dissemination of antibiotic-resistant pathogens. Furthermore, the isolation of infected or colonized patients is of paramount importance.

Global antimicrobial susceptibility surveillance systems should also focus on the prevalence of multidrug-resistant/pan-resistant organisms, rather than restricting to just resistance rates to individual antibiotics so that the global impact of this problem could be assessed.

\section{AUTHOR'S CONTRIBUTIONS}

All the authors have made substantial contributions to conception, design, acquisition of data, analysis, interpretation, drafting manuscript, and have given final approval of the version to be published.

\section{CONFLICTS OF INTEREST}

None declared (by all authors)

FUNDING

Nil.

\section{REFERENCES}

1. Grewal US, Bakshi R, Walia G, Shah PR. Antibiotic susceptibility profiles of non-fermenting gram-negative Bacilli at a tertiary care hospital in Patiala, India. Niger Postgrad Med J 2017;24:121-25.

2. Gore S, Pai C. Characterization and antibiotic susceptibility pattern of gram-negative non-fermenters in various clinical samples in tertiary care hospital of Navi Mumbai. Int J Curr Microbiol App Sci 2015;4:623-29.

3. Koneman EW. Color atlas and textbook of diagnostic microbiology. In: Koneman EW, Atlan SD, Dowell, Sommers HB, editors. The Non Fermentative Gram Negative Bacilli. $6^{\text {th }}$ ed. Philadelphia, PA: JB Lippincott Co.; 2006. p. 309-75.

4. Sharma M, Pant ND. Prevalence and in vitro antimicrobial susceptibility pattern of non-lactose fermenting gram-negative bacteria isolated in a tertiary care hospital in Kathmandu, Nepal. Asian J Biomed Pharm Sci 2017;7:13-6.

5. Kamalraj M, Sivashankari S, Thamarai S, Sastry AS. Study on non fermenting gram-negative Bacilli from various clinical samples in a tertiary care hospital. Int J Biol Med Res 2015;6:5230-35.

6. Juyal D, Prakash R, Shanakarnarayan SA, Sharma M, Negi V, Sharma N. Prevalence of non-fermenting gram-negative Bacilli and in vitro susceptibility pattern in a tertiary care hospital of Uttarakhand: A study from foothills of Himalayas. Saudi J Health Sci 2013;2:108-12.

7. Bhargava D, Kar S, Saha M. Prevalence of non-fermenter gramnegative Bacilli infection in tertiary care hospital in Birgunj, Nepal. Int J Curr Microbiol App Sci 2015;4:301-7.

8. Benachinmardi KK, Padmavathy M, Malini J, Nave-Neeth BV. Prevalence of non-fermenting Gram-negative Bacilli and their in vitro susceptibility pattern at a tertiary care teaching hospital. J Sci Soc 2014;41:162-6.

9. Collee JG, Marr W. Mackie and McCartney practical medical microbiology. In: Collee JG, Fraser AG, Marmion BP, Simmons A, editors. Culture of Bacteria. 14 ${ }^{\text {th }}$ ed. London: Churchill Livingstone; 2006. p. 113-29.

10. Collee JG, Miles RS, Watt B. Mackie and McCartney practical medical microbiology. In: Collee JG, Fraser AG, Marmion BP, Simmons A, editors. Tests for the Identification of Bacteria.14 ${ }^{\text {th }}$ ed. London: Churchill Livingstone; 2006. p. 131-49.

11. Clinical and Laboratory Standards Institute. Performance Standards for Antimicrobial Susceptibility Testing. 26 ${ }^{\text {th }}$ ed. Wayne, PA: Clinical and Laboratory Standards Institute; 2017.

12. Reddy R, Shirisha BK, Singh M, Kabra V. Isolation and identification of non-fermentative gram-negative Bacilli. Int J Curr Microbiol App Sci 2017:8:1003-10

13. Sajjad A, Javed I, Mushtaq S, Anwer MS. Frequency and susceptibility pattern of non-fermenter gram-negative Bacilli in a tertiary care hospital. Biomedica 2017;33:174-78.

14. Bohra R, Wadhwa R, Bala K. Isolation and characterization of lactose and non-lactose fermenting Bacteria from tertiary care hospital and their antimicrobial susceptibility test. Asian J Pharm Clin Res 2017;10:201-5.

15. Akbar M, Zahid M, Ali PA, Sthanadar AA, Shah M, Sthanadar IA, et al. Isolation and identification of multi-drug resistant strains of non-lactose fermenting Bacteria from clinical isolates. Open J Med Microbiol 2014;4:115-23.

16. Malini A, Deepa EK, Gokul BN, Prasad SR. Nonfermenting gramnegative Bacilli infections in a tertiary care hospital in Kolar, Karnataka. J Lab Physicians 2009;1:62-6.

17. Upgade A, Prabhu N, Gopi V, Soundararajan N. Current status of antibiotic-resistant non-fermentative gram-negative Bacilli among nosocomial infections. Adv Appl Sci Res 2012;3:738-42.

18. Nautiyal S, Jauhari S, Goel N, Mahawal BS. The current trend of nonfermenting gram-negative Bacilli in a tertiary care hospital in Dehradun, Uttarakhand. Int J Adv Res 2014;2:322-8.

19. Nazir A, Peerzada BY, Sana I. Spectrum of non-fermenting gramnegative Bacilli isolated from patients with bloodstream infections in a tertiary care hospital in North India. Int J Res Med Sci 2019;7:1762-66.

20. Gunasekar B, Shameembanu AS, Kalyani M. Non-fermenting gramnegative Bacilli: Phenotypic identification and correlation between biofilm formation and antibiotic susceptibility testing. Int J Res Pharm 2018:9:1229-34.

21. Mahajan DR, Neeraj D, Sarika D, Mahajan DB. Isolation and identification of non-fermenting gram-negative Bacilli in A tertiary care hospital. Sch J App Med Sci 2016;4:872-6.

22. Shah MH, Vaghela GM. Prevalence and antibiotic profile of nonfermenters at tertiary care hospital, Surat. Int J Biomed Adv Res 2018;9:316-8

23. Paul D, Borah AK. The emergence of non-fermenting gram-negative Bacilli as multi-drug resistant septicaemic pathogen in a tertiary hospital. Acta Sci Microbiol 2020;3:1-5.

24. De Francesco MA, Ravizzola G, Peroni L, Bonfanti C, Manca N. Prevalence of multidrug-resistant Acinetobacter baumannii and Pseudomonas aeruginosa in an Italian hospital. J Infect Public Health 2013;6:179-85.

25. Wang JT, Chen PC, Chang SC, Shiau YR, Wang HY, Lai JF, et al. Antimicrobial susceptibilities of Proteus mirabilis: A longitudinal nationwide study from the Taiwan surveillance of antimicrobial resistance (TSAR) program. BMC Infect Dis 2014;14:486.

26. Leulmi Z, Kandouli C, Benlabed K, Lezza A, Mihoubi I. Prevalence and evaluation of resistance to antibiotics of genera Proteus, Morganella and providencia isolates in university hospital of constantine, Algeria. Int J Adv Res 2014;2:220-7.

27. Bahashwan SA. Antimicrobial resistance patterns of Proteus isolates from clinical specimens. Eur Sci J 2013;9:188-202.

28. Dutta S, Rajendran K, Roy S, Chatterjee A, Dutta P, Nair GB, et al. Shifting serotypes, plasmid profile analysis, and antimicrobial resistance pattern of shigellae strains isolated from Kolkata, India during 1995-2000. Epidemiol Infect 2002;129:235-43.

29. Pazhani GP, Ramamurthy T, Mitra U, Bhattacharya SK, Niyogi SK. Species diversity and antimicrobial resistance of Shigella spp. Isolated between 2001 and 2004 from hospitalized children with diarrhea in Kolkata (Calcutta), India. Epidemiol Infect 2005;133:1089-95.

30. Puzari M, Sharma M, Chetia P. Emergence of antibiotic-resistant Shigella species: A matter of concern. J Infect Public Health 2018;11:451-4

31. Srinivasa H, Baijayanti M, Raksha Y. Magnitude of drug-resistant shigellosis: A report from Bangalore. Indian J Med Microbiol 2009;27:358-60.

32. Mamatha B, Rituparna C. Decreased susceptibility to antimicrobials among Shigella flexneri isolates in Manipal, South India-a 5-year hospital-based study. Southeast Asian J Trop Med Public Health 2012;43:47-51.

33. Jain PA, Kulkarni RD, Dutta S, Ganavali AS, Kalabhavi AS, Shetty PC, et al. Prevalence and antimicrobial profile of Shigella isolates in a tertiary care hospital of North Karnataka: A 12-year study. Indian J Med Microbiol 2020;38:101-8.

34. Liu J, Wang R, Fang M. Clinical and drug resistance characteristics of Providencia stuartii infections in 76 patients. J Int Med Res 2020;48:1-5.

35. Nagaveni S, Rajeshwari H, Oli A, Patil SA, Kelmani CR. The bioscan. eMagazine 2010;20:251-53.

36. Anuradha K, Meena AK, Lakshmi V. Isolation of Burkholderia pseudomallei from a case of septicemia: A case report. Indian J Med Microbiol 2003;21:129-32

37. Gupta V, Chhina D, Kaur A. Incidence of metallo-beta-lactamase (MBL) producing non fermenters isolated from respiratory samples in ICU patients. Int J Pharm Bio Sci 2013;4:580-5.

38. Hodiwala A, Dhoke R, Urhekar AD. Incidence of Metallo-betalactamase producing Pseudomonas, Acinetobacter, and enterobacterial isolates in hospitalized patients. Int J Pharm Bio Sci 2013;3:79-83.

39. Uma KR, Srinivasa RR, Suchismita S, Shashikala P, Kanungo R, 
Jayachandran S, et al. Phenotypic and genotypic assays for detecting the prevalence of Metallo-b-lactamases in the clinical isolates of Acinetobacter baumannii from a south Indian tertiary care hospital. J Med Microbiol 2009;58:430-5.

40. Kumar AV, Pillai VS, Dinesh K, Karim S. The phenotypic detection of carbapenemase in meropenem resistant acinetobacter calcoaceticusbaumannii complex in a tertiary care hospital in South India. J Clin Diagn Res 2011;5:223-6.

41. Sinha M, Srinivasan H, Macaden R. Antibiotic resistance profile and extended beta-lactamase (ESBL) production in Acinetobacter species. Indian J Pathol Microbial 2007;126;63-7.

42. O'Hara CM, Brenner FW, Miller JM. Classification, identification, and clinical significance of Proteus, Providencia, and Morganella. Clin Microbiol Rev 2000;13:534-46.

43. Feglo PK, Gbedema SY, Quay SN, Adu-Sarkodie Y, Opoku-Okrah C. Occurrence, species distribution, and antibiotic resistance of Proteus isolates: A case study at the Komfo Anokye teaching hospital (KATH) in Ghana. Int J Pharma Sci Res 2010;1:347-52.

44. Mirzaei A, Habibi M, Bouzari S, Karam MR. Characterization of antibiotic-susceptibility patterns, virulence factor profiles and clonal relatedness in proteus mirabilis isolates from patients with urinary tract infection in Iran. Infect Drug Resist 2019;12:3967-79.

45. Swenson JM, Hindler JA, Peterson LR. Manual of clinical microbiology. In: Murray PR, editor. Special Phenotypic Methods for Detecting Antibacterial Resistance. $7^{\text {th }}$ ed. Washington, DC: American Society for Microbiology; 1999. p. 1563-77.

46. Fass RJ, Barnishan J, Ayers LW. The emergence of bacterial resistance to imipenem and ciprofloxacin in a university hospital. J Antimicrob Chemother 1995;36:343-53.
47. Mamatha B, Pusapati BR, Rituparna C. Changing patterns of antimicrobial susceptibility of Shigella serotypes isolated from children with acute diarrhea in Manipal, South India, A 5-year study. Southeast Asian J Trop Med Public Health 2007;38:863-66.

48. World Health Organization. Guidelines for the Control of Shigellosis, Including Epidemics Due to Shigella dysenteriae Type 1. Geneva: World Health Organization; 2005. Available from: http://www. whqlibdoc. who.int/publications/2005/9241592330.pdf. [Last accessed on 2019 Jul 17].

49. Mehta A, Singh VP, Sharma D. Antibiotic susceptibility pattern of blood culture isolates of Enteric fever pathogens in a tertiary care center-a retrospective study. Int J Res Health Sci 2018;6:9-15.

50. Choudhary A, Gopalakrishnan R, Nambi PS, Ramasubramanian V, Ghafur KA, Thirunarayan MA. Antimicrobial susceptibility of Salmonella enterica serovars in a tertiary care hospital in southern India. Indian J Med Res 2013;137:800-2.

51. Singhal L, Gupta PK, Kale P, Gautam V, Ray P. Trends in antimicrobial susceptibility of Salmonella Typhi from North India. Ind J Med Microbiol 2014;32:149-52.

52. Kumar Y, Sharma A, Maniet KR. Antibiogram profile of Salmonella enterica serovar typhi in India-a two year study. Trop Life Sci Res 2013;24:45-54.

53. Gurung B, Pandey S, Shah DK, Mandal MK, Bhatta B. Antibiogram pattern of Salmonella in blood samples of enteric fever patients at Lalitpur, Nepal. Asian Pac J Trop Dis 2017;7:21-4.

54. Mehta A, Tukaram P. Detection and characterization of Metallo- $\beta$ lactamases producing Pseudomonas aeruginosa clinical isolates at a tertiary care hospital of Bhopal, India. Int J Res Med Sci 2016;4:4084-88. 